\title{
Classical limit of master equation for harmonic oscillator coupled to oscillator bath with separable initial conditions
}

\author{
Subhashish Banerjee ${ }^{1, *}$ and Abhishek Dhar ${ }^{1, t}$ \\ ${ }^{1}$ Raman Research Institute, Bangalore 560080
}

(Dated: July 27, 2018)

\begin{abstract}
The equation for the Wigner function describing the reduced dynamics of a single harmonic oscillator, coupled to an oscillator bath, was obtained by Karrlein and Grabert [Phys. Rev. E 55, 153 (1997)]. It was shown that for some special correlated initial conditions the equation reduces, in the classical limit, to the corresponding classical Fokker-Planck equation obtained by Adelman [J. Chem Phys. 64, 124 (1976)]. However for separable initial conditions the Adelman equations were not recovered. We resolve this problem by showing that, for separable initial conditions, the classical Langevin equation obtained from the oscillator bath model is somewhat different from the one considered by Adelman. We obtain the corresponding Fokker-Planck equation and show that it exactly matches the classical limit of the equation for the Wigner function obtained from the master equation for separable initial conditions. We also discuss why the special correlated initial conditions correspond to Adelman's solution.
\end{abstract}

PACS numbers: 05.30.-d, 05.40.-a, 05.70.Ln 


\section{INTRODUCTION}

The concept of 'open' quantum systems is a ubiquitous one in that any real system is never fully isolated. It is usually in contact with a larger system, constituting its environment, which effects its dynamics. Ford, Kac and Mazur ${ }^{1}$ suggested the first microscopic model describing dissipative effects. They considered a system which was coupled to a bath modelled by a collection of an infinite number of harmonic oscillators. Interest in quantum dissipation was intensified by the work of Caldeira and Leggett ${ }^{2}$ who used the influence functional approach, developed by Feynman and Vernon ${ }^{3}$, to discuss quantum Brownian motion. They considered the case where the system and its environment were initially uncorrelated, the so called separable initial condition. This was generalized to the situation where initial correlations exist between the system and its environment by Hakim and Ambegaokar ${ }^{4}$, Smith and Caldeira ${ }^{5}$, Grabert, Schramm and Ingold ${ }^{6}$, Chen, Lebowitz and Liverani $\underline{7}^{7}$ and Banerjee and Ghosh ${ }^{8}$ among others. The master equation for the quantum Brownian motion of a harmonic oscillator in a bosonic bath of harmonic oscillators was obtained by Haake and Reibold ${ }^{9}$ and later by $\mathrm{Hu}, \mathrm{Paz}$ and Zhang ${ }^{10}$ who used path integral methods in their derivation. A derivation of the Wigner transform of this equation was also given by Halliwell and $\mathrm{Yu}^{11}$ and a solution of this Fokker-Planck equation with time dependent coefficients was given by Ford and O'Connel12.

In a very interesting paper Karrlein and Grabert ${ }^{13}$ studied the master equation for the reduced density matrix of the system for generalized correlated initial conditions. They showed that in general it is not possible to obtain a Liouvillean operator for the master equation of the reduced dynamics of the system under the influence of its environment. However for some specialized correlated initial conditions like the one discussed by Hakim and Ambegaokar ${ }^{4}$, called the thermal initial condition, and for the case of the time evolution of equilibrium correlation functions ${ }^{6}$, it is possible to obtain the Liouvillean to describe the reduced system dynamics. In both these cases Karrlein and Grabert ${ }^{13}$ obtained the FokkerPlanck equation from the Wigner transform of the master equation. They found that in the classical limit, these equations reduced exactly to the corresponding equation obtained by Adelman ${ }^{14}$ for the dissipative dynamics of a classical harmonic oscillator driven by a Gaussian non-Markovian noise. They then considered the case of separable initial conditions and found that in the classical limit the corresponding Fokker-Planck equation did not reduce 
to the Adelman equation. We find this a very intriguing as well as an interesting point and the main purpose of this paper is to look at this point more closely. This is also important to understand in view of the fact that factorizing initial conditions are known to have some problematic features $\frac{6.7}{}$ associated with the sudden switching on of the coupling.

The plan of this paper is as follows. In Sec. II we obtain the Langevin equation for the system consisting of a harmonic oscillator coupled to a bath of harmonic oscillators with which it is initially uncorrelated. We indicate that our treatment is valid in the classical as well as the quantum regime. In Sec. III we obtain the Fokker-Planck equation from the Langevin equation derived in Sec. II in the classical regime. When we compare our equation with the one used by Adelman ${ }^{14}$ we find that our equation has an additional term. This additional term is the crux which enables us to resolve the issue. The Fokker-Planck equation obtained from the Langevin equation with this additional term is the exact classical limit of the corresponding equation in the quantum regime obtained by Karrlein and Grabert ${ }^{13}$. In Sec. IV we discuss why the Langevin equation used by Adelman would be the correct classical equation describing a correlated initial state. In Sec. V we discuss our conclusions.

\section{LANGEVIN EQUATION}

We consider a single harmonic oscillator described by the phase space variables $(x, p)$ and with a natural frequncy $\omega_{0}$. The oscillator is coupled to a heat bath modelled by $N$ independent oscillators described by $\left\{X_{\alpha}, P_{\alpha}\right\}$ and which have frequencies $\left\{\omega_{\alpha}\right\}$. To generate dissipation one eventually takes the limit $N \rightarrow \infty$. The full Hamiltonian is taken to be:

$$
H=\frac{p^{2}}{2}+\frac{\omega_{0}^{2} x^{2}}{2}+\sum_{\alpha=1}^{N}\left[\frac{P_{\alpha}^{2}}{2}+\frac{\omega_{\alpha}^{2} X_{\alpha}^{2}}{2}\right]-\sum_{\alpha=1}^{N} c_{\alpha} x X_{\alpha} .
$$

The last term represents the coupling between the system and the bath. Introducing the shifted frequency $\omega^{2}=\omega_{0}^{2}-\sum_{\alpha} c_{\alpha}^{2} / \omega_{\alpha}^{2}$, we can rewrite Eq. (11) in the following equivalent form

$$
H=\frac{p^{2}}{2}+\frac{\omega^{2} x^{2}}{2}+\sum_{\alpha=1}^{N}\left[\frac{P_{\alpha}^{2}}{2}+\frac{\omega_{\alpha}^{2}\left(X_{\alpha}-\frac{c_{\alpha} x}{\omega_{\alpha}^{2}}\right)^{2}}{2}\right] .
$$


Let us now write the equations of motion for the system and bath variables. These are given by:

$$
\begin{aligned}
\ddot{x} & =-\omega_{0}^{2} x+\sum_{\alpha} c_{\alpha} X_{\alpha}, \\
\ddot{X}_{\alpha} & =-\omega_{\alpha}^{2} X_{\alpha}+c_{\alpha} x .
\end{aligned}
$$

The solution of the equations of motion of the reservoir variables gives

$$
X_{\alpha}(t)=\cos \left(\omega_{\alpha} t\right) X_{\alpha}(0)+\frac{\sin \left(\omega_{\alpha} t\right)}{\omega_{\alpha}} \dot{X}_{\alpha}(0)+\int_{0}^{t} d t^{\prime} \frac{\sin \omega_{\alpha}\left(t-t^{\prime}\right)}{\omega_{\alpha}} c_{\alpha} x\left(t^{\prime}\right) .
$$

Plugging these into the system's equation of motion Eq. (3) we get the following Langevin equations of motion:

$$
\begin{aligned}
\ddot{x} & =-\omega_{0}^{2} x+\int_{0}^{t} d t^{\prime} \frac{\partial \gamma\left(t-t^{\prime}\right)}{\partial t^{\prime}} x\left(t^{\prime}\right)+\eta(t), \\
\text { where } \gamma(t) & =\sum_{\alpha} \frac{c_{\alpha}^{2}}{\omega_{\alpha}^{2}} \cos \left(\omega_{\alpha} t\right) \\
\eta(t) & =\sum_{\alpha} c_{\alpha}\left[\cos \left(\omega_{\alpha} t\right) X_{\alpha}(0)+\frac{\sin \left(\omega_{\alpha} t\right)}{\omega_{\alpha}} \dot{X}_{\alpha}(0)\right] .
\end{aligned}
$$

Note that the discussion till now is valid both clasically and quantum mechanically. In the quantum case the variables correspond to operators in the Heisenberg representation. We now use the fact that at $t=0$ the separable initial conditions imply that the reservoir (isolated) is initially in equilibrium and hence we know the statistical properties of the bath variables $\left\{X_{\alpha}(0), \dot{X}_{\alpha}(0)\right\}$. In the quantum case we get

$$
\begin{aligned}
& \left\langle\omega_{\alpha}^{2} X_{\alpha}^{2}(0)\right\rangle=\left\langle\dot{X}_{\alpha}^{2}(0)\right\rangle=\frac{\hbar \omega_{\alpha}}{2} \operatorname{coth} \frac{\hbar \omega_{\alpha}}{2 k_{B} T}, \\
& \left\langle\left[X_{\alpha}(0) \dot{X}_{\alpha}(0)+\dot{X}_{\alpha}(0) X_{\alpha}(0)\right]\right\rangle=0 .
\end{aligned}
$$

In the classical case we get, using equipartition, $\left\langle\omega_{\alpha}^{2} X_{\alpha}^{2}\right\rangle=\left\langle\dot{X}_{\alpha}^{2}\right\rangle=k_{B} T$ and $\left\langle X_{\alpha} \dot{X}_{\alpha}\right\rangle=0$ and these also follow from Eq. (9) if we take the classical limit $\hbar \omega_{\alpha} /\left(k_{B} T\right) \rightarrow 0$. From these properties of the bath one can work out the correlations of the noise $\eta(t)$ in Eq. (8) . We get $\left(\right.$ for $\left.t, t^{\prime}>0\right)$

$$
\begin{aligned}
\langle\eta(t)\rangle & =0 \\
\frac{1}{2}\left\langle\eta(t) \eta\left(t^{\prime}\right)+\eta\left(t^{\prime}\right) \eta(t)\right\rangle & =\hbar K^{\prime}\left(t-t^{\prime}\right) \\
\text { where } \quad K^{\prime}(t) & =\sum_{\alpha} \frac{c_{\alpha}^{2}}{2 \omega_{\alpha}} \cos \omega_{\alpha} t \operatorname{coth} \frac{\hbar \omega_{\alpha}}{2 k_{B} T} .
\end{aligned}
$$


Here we have used the notation in Ref. 6. 13]. The classical limit of Eq. (10) gives the usual fluctuation-dissipation relation

$$
\left\langle\eta(t) \eta\left(t^{\prime}\right)\right\rangle=k_{B} T \gamma\left(t-t^{\prime}\right)
$$

\section{FOKKER-PLANCK EQUATION FOR THE CLASSICAL LANGEVIN EQUA- TION}

In this section we focus on the classical Langevin equation which is described by Eqs. (66), (12). First we note that, after a partial integration, we can rewrite Eq. (6) in the following form:

$$
\ddot{x}=-\omega^{2} x-\gamma(t) x(0)-\int_{0}^{t} d t^{\prime} \gamma\left(t-t^{\prime}\right) \dot{x}\left(t^{\prime}\right)+\eta(t) .
$$

This differs from the equation considered by Adelman 14 by the initial condition dependent term $-\gamma(t) x(0)$, the so called initial slip term 15,16 which, as we will see, is crucial in getting the correct Fokker-Planck equation. Starting from the above Langevin equation or its equivalent form Eq. (6) we get, following Adelman, the corresponding Fokker-Planck equation.

The general solution of Eq. (6) is given by:

$$
x(t)=H(t) x(0)+G(t) p(0)+\int_{0}^{t} d t^{\prime} G\left(t-t^{\prime}\right) \eta\left(t^{\prime}\right),
$$

where $H(t)$ and $G(t)$ are solutions of the homogeneous part of Eq. (6) for the initial condi-

tions $H(0)=1, \dot{H}(0)=0$ and $G(0)=0, \dot{G}(0)=1$ respectively, and $p(t)=\dot{x}(t)$. Taking a Laplace transform of the homogeneous equation we immediately get:

$$
\begin{aligned}
& \hat{G}(z)=\frac{1}{z^{2}+\omega^{2}+z \hat{\gamma}(z)}, \\
& \hat{H}(z)=\frac{z}{z^{2}+\omega^{2}+z \hat{\gamma}(z)},
\end{aligned}
$$

where $\hat{G}(z), \hat{H}(z)$ and $\hat{\gamma}(z)$ are the Laplace transforms of $G(t), H(t)$ and $\gamma(t)$ respectively. We note that $G(t)$ is the same Green's function, denoted by $\chi_{u}(t)$, in Adelman's $\frac{14}{}$ paper. However $H(t)$ is different from Adelman's $\chi_{x}$, which we will here denote by $F(t)$, and which is given by

$$
F(t)=1-\omega^{2} \int_{0}^{t} d t^{\prime} G\left(t^{\prime}\right)
$$


From the form of the Laplace transforms $\hat{G}, \hat{H}$ and from Eq. (17) it is also easy to get the relations

$$
\dot{G}(t)=H(t) \text { and } \quad \dot{F}(t)=-\omega^{2} G(t)
$$

From the general solution for the position we get for the momentum

$$
p(t)=\dot{H}(t) x(0)+\dot{G}(t) p(0)+\int_{0}^{t} d t^{\prime} \dot{G}\left(t-t^{\prime}\right) \eta\left(t^{\prime}\right) .
$$

Let us use the notation $\left\{x_{1}, x_{2}\right\}=\{x, p\}$. The fluctuations about the mean position and momentum are given by:

$$
\begin{aligned}
& y_{1}(t)=x_{1}(t)-[H(t) x(0)+G(t) p(0)]=\int_{0}^{t} d t^{\prime} G\left(t-t^{\prime}\right) \eta\left(t^{\prime}\right), \\
& y_{2}(t)=x_{2}(t)-[\dot{H}(t) x(0)+\dot{G}(t) p(0)]=\int_{0}^{t} d t^{\prime} \dot{G}\left(t-t^{\prime}\right) \eta\left(t^{\prime}\right) .
\end{aligned}
$$

In the classical case, since $\eta(t)$ depends linearly on $X_{\alpha}(0)$ and $\dot{X}_{\alpha}(0)$ which have a Gaussian distribution, it follows that $\eta(t)$ is also Gaussian. Hence the distribution of $\left\{y_{1}, y_{2}\right\}$ at any time will also be Gaussian and is completely specified by the following variances, obtained from Eqs. (12), (20):

$$
\begin{aligned}
& A_{11}(t)=\left\langle y_{1}^{2}\right\rangle=k_{B} T \int_{0}^{t} d t^{\prime} \int_{0}^{t} d t^{\prime \prime} G\left(t^{\prime}\right) G\left(t^{\prime \prime}\right) \gamma\left(t^{\prime}-t^{\prime \prime}\right), \\
& A_{22}(t)=\left\langle y_{2}^{2}\right\rangle=k_{B} T \int_{0}^{t} d t^{\prime} \int_{0}^{t} d t^{\prime \prime} \dot{G}\left(t^{\prime}\right) \dot{G}\left(t^{\prime \prime}\right) \gamma\left(t^{\prime}-t^{\prime \prime}\right), \\
& A_{12}(t)=A_{21}(t)=\left\langle y_{1} y_{2}\right\rangle=k_{B} T \int_{0}^{t} d t^{\prime} \int_{0}^{t} d t^{\prime \prime} G\left(t^{\prime}\right) \dot{G}\left(t^{\prime \prime}\right) \gamma\left(t^{\prime}-t^{\prime \prime}\right) .
\end{aligned}
$$

Taking time-derivatives and after some manipulations one can get the following results for the variances:

$$
\begin{aligned}
& \dot{A}_{11}=-2 k_{B} T\left(G \dot{G}+\frac{F \dot{F}}{\omega^{2}}\right), \\
& A_{11}=-k_{B} T\left(G^{2}+\frac{F^{2}-1}{\omega^{2}}\right), \\
& \dot{A}_{22}=-2 k_{B} T\left(\dot{G} \ddot{G}+\omega^{2} G \dot{G}\right), \\
& A_{22}=-k_{B} T\left(\omega^{2} G^{2}+\dot{G}^{2}-1\right), \\
& \dot{A}_{12}=k_{B} T\left(\frac{d}{d t}(G F)-\frac{d}{d t}(G \dot{G})\right), \\
& A_{12}=k_{B} T(G F-G \dot{G}) .
\end{aligned}
$$

These are of course identical to the results of Adelman since the variances involve exactly the same Green's function. However the mean values $\langle x(t)\rangle,\langle p(t)\rangle$ are different and we will 
now show that this leads to a different Fokker-Planck equation. The phase space probability distribution function for $\{x, p\}$ is thus

$$
P(x, p, t \mid x(0), p(0), 0)=\frac{1}{2 \pi(\operatorname{det}[\mathbf{A}])^{1 / 2}} e^{-y_{i} A_{i j}^{-1} y_{j} / 2} .
$$

Following Adelman, let us define the following matrix:

$$
\mathbf{T}^{-1}(t)=\left(\begin{array}{cc}
H(t) & G(t) \\
\dot{H}(t) & \dot{G}(t)
\end{array}\right)
$$

and new variables $\left\{q_{1}, q_{2}\right\}$ through the transformation

$$
\left(\begin{array}{l}
q_{1}(t) \\
q_{2}(t)
\end{array}\right)=\mathbf{T}(t)\left(\begin{array}{l}
x_{1}(t) \\
x_{2}(t)
\end{array}\right) .
$$

Clearly we then have $q_{1}(0)=x(0), q_{2}(0)=p(0)$. In terms of these new variables the probability density $P$ takes the following form:

$$
\begin{aligned}
\tilde{P}\left(q_{1}, q_{2}, t \mid x(0), p(0), 0\right) & =P\left(x_{1}, x_{2}, t \mid x(0), p(0), 0\right) \\
& =\frac{1}{2 \pi(\operatorname{det}[\mathbf{A}])^{1 / 2}} e^{-\left[q_{i}-q_{i}(0)\right] B_{i j}^{-1}\left[q_{j}-q_{j}(0)\right] / 2} \\
\text { where } \quad \mathbf{B} & =\mathbf{T A T}^{T} .
\end{aligned}
$$

Taking time and space derivatives of $\tilde{P}$ gives the following relations:

$$
\begin{aligned}
\frac{\partial \tilde{P}}{\partial t} & =-\frac{1}{2} \frac{d}{d t} \ln \operatorname{det}[\mathbf{A}] \tilde{P}-\frac{1}{2} \dot{B}_{l m}^{-1}\left[q_{l}-q_{l}(0)\right]\left[q_{m}-q_{m}(0)\right] \tilde{P} \\
\frac{1}{2} \frac{\partial^{2} \tilde{P}}{\partial q_{l} \partial q_{m}} \dot{B}_{l m} & =-\frac{1}{2} B_{l m}^{-1} \dot{B}_{l m} \tilde{P}+\frac{1}{2} B_{l i}^{-1} B_{m j}^{-1} \dot{B}_{l m}\left[q_{i}-q_{i}(0)\right]\left[q_{j}-q_{j}(0)\right] \tilde{P} \\
& =-\frac{1}{2} \frac{d}{d t} \ln \operatorname{det}[\mathbf{B}] \tilde{P}-\frac{1}{2} \dot{B}_{l m}^{-1}\left[q_{l}-q_{l}(0)\right]\left[q_{m}-q_{m}(0)\right] \tilde{P}
\end{aligned}
$$

where the last line follows from using the following identities for the symmetric matrix $\mathbf{B}$ :

$$
\begin{aligned}
\frac{d}{d t} \operatorname{det}[\mathbf{B}] & =\frac{\partial \operatorname{det}[\mathbf{B}]}{\partial B_{l m}} \dot{B}_{l m}=\operatorname{det}[\mathbf{B}] B_{l m}^{-1} \dot{B}_{l m} \\
\dot{\mathbf{B}}^{-1} & =-\mathbf{B}^{-1} \dot{\mathbf{B B}}^{-1}
\end{aligned}
$$

We will also need the following result:

$$
\frac{\partial \tilde{P}}{\partial t}=\frac{\partial P}{\partial t}+\dot{\mathbf{T}}_{l i}^{-1} \mathbf{T}_{i m} x_{m} \frac{\partial P}{\partial x_{l}}
$$


Using Eqs. (27), (28), (29) and transforming from the variables $\left\{q_{1}, q_{2}\right\}$ to $\left\{x_{1}, x_{2}\right\}$ we finally get:

$$
\frac{\partial P}{\partial t}=-\left[\dot{\mathbf{T}}^{-1} \mathbf{T}\right]_{l m} x_{m} \frac{\partial P}{\partial x_{l}}-\frac{d}{d t} \ln \Delta P+\frac{1}{2}\left[\mathbf{T}^{-1} \dot{\mathbf{B}} \mathbf{T}^{-1}\right]_{l m} \frac{\partial^{2} P}{\partial x_{l} \partial x_{m}},
$$

where $\Delta=\operatorname{det}\left[\mathbf{T}^{-1}\right]=\dot{G}^{2}-G \ddot{G}$. We work out the explicit forms of the various matrices and get:

$$
\begin{aligned}
\mathbf{T}^{-1} \dot{\mathbf{T}} & =-\dot{\mathbf{T}}^{-1} \mathbf{T}=\left(\begin{array}{cc}
0 & -1 \\
\gamma_{q} & \gamma_{p}
\end{array}\right), \\
\mathbf{T}^{-1} \dot{\mathbf{B}} \mathbf{T}^{-1} T & =\dot{\mathbf{A}}+\mathbf{T}^{-1} \dot{\mathbf{T}} \mathbf{A}+\left(\mathbf{T}^{-1} \dot{\mathbf{T}} \mathbf{A}\right)^{T} \\
& =\left(\begin{array}{cc}
\dot{A}_{11}-2 A_{12} & \dot{A}_{12}-A_{22}+\gamma_{q} A_{11}+\gamma_{p} A_{12} \\
\dot{A}_{12}-A_{22}+\gamma_{q} A_{11}+\gamma_{p} A_{12} & \dot{A}_{22}+2 \gamma_{q} A_{12}+2 \gamma_{p} A_{22}
\end{array}\right), \\
\text { where } \gamma_{q} & =\frac{\ddot{G}^{2}-\dot{G} \ddot{G}}{\dot{G}^{2}-G \ddot{G}}, \\
\gamma_{p} & =\frac{G \ddot{G}-\dot{G} \ddot{G}}{\dot{G}^{2}-G \ddot{G}}=-\frac{d}{d t} \ln \Delta .
\end{aligned}
$$

From Eq. (22) we get $\dot{A}_{11}=2 A_{12}$. Using this and expanding out Eq. (30) gives:

$$
\begin{aligned}
\frac{\partial P(x, p, t \mid x(0), p(0), 0)}{\partial t} & =-p \frac{\partial P}{\partial x}+\gamma_{q} x \frac{\partial P}{\partial p}+\gamma_{p} \frac{\partial(p P)}{\partial p}+D_{p} \frac{\partial^{2} P}{\partial p^{2}}+D_{q} \frac{\partial^{2} P}{\partial x \partial p} \\
\text { where } D_{q} & =\frac{1}{2} \ddot{A}_{11}-A_{22}+\gamma_{q} A_{11}+\frac{\gamma_{p}}{2} \dot{A}_{11} \\
D_{p} & =\frac{1}{2} \dot{A}_{22}+\frac{\gamma_{q}}{2} \dot{A}_{11}+\gamma_{p} A_{22}
\end{aligned}
$$

From Eq. (17) and Eq. (111) it is clear that in the classical limit:

$$
K^{\prime}(t) \rightarrow \frac{k_{B} T}{\hbar} \gamma(t)
$$

which implies that the classical limits of the functions $K_{q}(t)$ and $K_{p}(t)$ in Ref. [13] is:

$$
\hbar K_{q}(t) \rightarrow A_{11}, \quad \hbar K_{p}(t) \rightarrow A_{22}
$$

With this we immediately see that the coefficients of the equation for the Wigner function, given by Eq. (89) in Ref. [13], reduces in the classical limit, to those in the Fokker-Planck equation given by Eq. (36). 


\section{CORRELATED EQUILIBRIUM INITIAL CONDITIONS}

It is possible to understand the precise reason for the observed correspondence between the Adelman results and the quantum Fokker-Planck equation for the correlated initial conditions considered in [13]. We will now show that the Langevin equation used by Adelman also follows from the same microscopic model if, instead of choosing a separable initial condition, we take a correlated initial state which is prepared by making measurements on an equilibrium state of the coupled system and bath. We first make some comments on the physical significance of the special correlated initial conditions that have been studied in the quantum case. The two initial conditions correspond to (i) making measurements of the position variable of the system at time $t=0$ on the equilibrium state, (ii) computing time dependent correlation functions of various system variables, again in an equilibrium state. Especially it is to be noted that the measurements are made on the equilibrium state of the fully coupled system and bath. Classically if we want to describe a similar situation, then the corresponding Langevin equation should decribe the time evolution of the system's phase

space variables $\{x, p\}$ given that an initial measurement, in the initial equilibrium state of the coupled system and bath, gave the values $\{x(0), p(0)\}$.

Starting from the same model of reservoir with the full Hamiltonian given by Eq. (11) or equivalently Eq. (2) we again get an equation in the form of Eq. (13) but the noise correlations are not given by Eq. (12). At $t=0$ the bath and system variables are correlated, hence $\left\langle x(0) X_{\alpha}(0)\right\rangle \neq 0$ and the bath correlations are no longer give by Eq. (9) or its classical counterpart. A simplification now occurs if we redefine the noise $\frac{17}{2}$ and write Eq. (13) in the following form:

$$
\ddot{x}=-\omega^{2} x-\int_{0}^{t} d t^{\prime} \gamma\left(t-t^{\prime}\right) \dot{x}\left(t^{\prime}\right)+\xi(t),
$$

where $\xi(t)=\eta(t)-\gamma(t) x(0)$

$$
=\sum_{\alpha} c_{\alpha}\left\{\cos \left(\omega_{\alpha} t\right)\left[X_{\alpha}(0)-\frac{c_{\alpha}}{\omega_{\alpha}^{2}} x(0)\right]+\frac{\sin \left(\omega_{\alpha} t\right)}{\omega_{\alpha}} \dot{X}_{\alpha}(0)\right\} .
$$

The statistical properties of the noise should be evaluated by averaging over the bath variables. Following the discussion in the previous paragraph it is clear that the distribution of the bath variables is given by the conditional probability of $\left\{X_{\alpha}(0), P_{\alpha}(0)\right\}$ given that an initial measurement, in the initial equilibrium state, on the system gave the values $\{x(0), p(0)\}$. 
From the form of the Hamiltonian Eq. (2), we get for the conditional distribution:

$$
\begin{aligned}
\operatorname{Pr}\left(\left\{X_{\alpha}(0), P_{\alpha}(0)\right\}\right) & =\frac{e^{-\beta H_{B}}}{Z_{B}} \\
\text { where } H_{B} & =\sum_{\alpha=1}^{N}\left[\frac{P_{\alpha}^{2}(0)}{2}+\frac{\omega_{\alpha}^{2}\left(X_{\alpha}(0)-\frac{c_{\alpha} x(0)}{\omega_{\alpha}^{2}}\right)^{2}}{2}\right], \\
Z_{B} & =\int \prod_{\alpha} d X_{\alpha}(0) d P_{\alpha}(0) e^{-\beta H_{B}} .
\end{aligned}
$$

Averaging the bath variables over this distribution we then get:

$$
\begin{aligned}
\left\langle\left[X_{\alpha}(0)-\frac{c_{a}}{\omega_{\alpha}^{2}} x(0)\right] \dot{X}_{\alpha}(0)\right\rangle & =0 \\
\left\langle\omega_{\alpha}^{2}\left[X_{\alpha}(0)-\frac{c_{a}}{\omega_{\alpha}^{2}} x(0)\right]^{2}\right\rangle & =k_{B} T \\
\left\langle\dot{X}_{\alpha}^{2}(0)\right\rangle & =k_{B} T .
\end{aligned}
$$

Using these we then find that $\xi(t)$ is again a Gaussian stationary process with exactly the same correlations as obtained for $\eta(t)$ for separable initial conditions. Thus

$$
\begin{aligned}
\langle\xi(t)\rangle & =0 \\
\left\langle\xi(t) \xi\left(t^{\prime}\right)\right\rangle & =k_{B} T \gamma\left(t-t^{\prime}\right)
\end{aligned}
$$

The Langevin equation given by Eq. (39) with the noise given by Eq. (42) is the starting equation of Adelman ${ }^{14}$. Hence we have shown that the Langevin equation of Adelman is the correct classical equation desribing a correlated initial state.

\section{DISCUSSION AND CONCLUSIONS}

In this paper we have taken up the issue of the classical limit of the quantum FokkerPlanck equation for a harmonic oscillator linearly coupled to a bath of harmonic oscillators wherein the system and bath are initially uncorrelated. Karrlein and Grabert 13 obtained the quantum Fokker-Planck equation for some special correlated as well as separable initial conditions. Two kinds of correlated initial conditions were studied, the so called thermal initial condition ${ }^{4}$ and those describing the dynamics of equilibrium correlation functions ${ }^{6}$. On taking the classical limit of these equations they found that they exactly reduced to Adelman's classical Fokker-Planck equation ${ }^{14}$. However for separable initial conditions they

found that the classical limit of their equation did not give Adelman's equation. It was suggested that this indicated a problem with separable initial conditions. 
We have found a simple resolution to this somewhat intriguing point. We derived the classical Langevin equations of motion corresponding to the system of a single oscillator coupled to a bath of harmonic oscillators and with separable initial conditions. This equation differs from the one used by Adelman ${ }^{14}$ by an extra piece $-\gamma(t) x(0)$ which depends on initial conditions. We then derived the Fokker-Planck equation for this Langevin equation and found it to be the exact classical limit of the quantum equation of Karrlein and Grabert ${ }^{13}$ for separable initial conditions. We have also shown why for the case of correlated initial conditions the Wigner function equation of the reduced system dynamics in the quantum regime ${ }^{13}$ reduces to the Adelman equation in the classical limit. We have shown that the Langevin equation used by Adelman corresponds to the situation where the initial state is the equilibrium state of the coupled system and reservoir. Thus the basic point is that correlated and uncorrelated initial conditions lead to different classical Langevin equations and hence to different Fokker-Planck equations. In conclusion we have shown that for the case of a single harmonic oscillator coupled to a bath of harmonic oscillators there is an exact correspondence between the classical Fokker-Planck equation and the quantum equations for the Wigner function both for some special correlated as well as uncorrelated initial conditions.

* Electronic address: subhashishb@rri.res.in

$\dagger$ Electronic address: dabhi@rri.res.in

1 G. W. Ford, M. Kac and P. Mazur, J. Math. Phys 6, 504 (1965).

2 A. O. Caldeira and A. J. Leggett, Physica A 121, 587 (1983).

3 R. P. Feynman and F. L. Vernon, Ann. Phys. (N.Y.) 24, 118 (1963).

4 V. Hakim and V. Ambegaokar, Phys. Rev. A 32, 423 (1985).

5 C. M. Smith and A. O. Caldeira, Phys. Rev. A 36, 3509 (1987); ibid 41, 3103 (1990).

6 H. Grabert, P. Schramm and G. L. Ingold, Phys. Rep. 168, 115 (1988).

7 Y.-C. Chen, J. L. Lebowitz and C. Liverani, Phys. Rev. B 40, 4664 (1989).

8 S. Banerjee and R. Ghosh, Phys. Rev E 67, 056120 (2003).

9 F. Haake and R. Reibold, Phys. Rev A 32, 2462 (1985).

10 B. L. Hu, J. P. Paz and Y. Zhang, Phys. Rev D 45, 2843 (1992). 
11 J. J. Halliwell and T. Yu, Phys. Rev D 53, 2012 (1996).

12 G. W. Ford and R. F. O'Connell, Phys. Rev. D 64, 105020 (2001).

13 R. Karrlein and H. Grabert, Phys. Rev. E 55, 153 (1997).

14 S. A. Adelman, J. Chem. Phys. 64, 124 (1976).

15 G. L. Ingold, Lect. Notes Phys. 611, 1 (2002).

16 P. Hanggi, Lect. Notes Phys. 484, 15 (1997).

17 P. Hanggi and G. L. Ingold, Chaos 15, 026105 (2005). 